\title{
Extended virtual element method for the torsion problem of cracked prismatic beams
}

\author{
Andrea Chiozzi • Elena Benvenuti
}

Received: date / Accepted: date

\begin{abstract}
In this paper, we investigate the capability of the recently proposed extended virtual element method (X-VEM) to efficiently and accurately solve the problem of a cracked prismatic beam under pure torsion, mathematically described by the Poisson equation in terms of a scalar stress function. This problem is representative of a wide class of elliptic problems for which classic finite element approximations tend to converge poorly, due to the presence of singularities. The $\mathrm{X}-\mathrm{VEM}$ is a stabilized Galerkin formulation on arbitrary polygonal meshes derived from the virtual element method (VEM) by augmenting the standard virtual element space with an additional contribution that consists of the product of virtual nodal basis functions with a suitable enrichment function. In addition, an extended projector that maps functions lying in the extended virtual element space onto linear polynomials and the enrichment function is employed. Convergence of the method on both quadrilateral and polygonal meshes for the cracked beam torsion problem is studied by means of numerical experiments. The computed results affirm the sound accuracy of the method and demonstrate a significantly improved convergence rate, both in terms of energy and stress intensity factor, when compared to standard finite element method (FEM) and VEM.
\end{abstract}

Keywords Virtual Element Method · Extended Virtual Element Method - Partition of Unity Method .

\footnotetext{
A. Chiozzi (凶)

Department of Engineering, University of Ferrara

Via Saragat, 1 - 44122 Ferrara, Italy

E-mail: andrea.chiozzi@unife.it

E. Benvenuti

Department of Engineering, University of Ferrara

Via Saragat, 1 - 44122 Ferrara, Italy

E-mail: elena.benvenuti@unife.it
}

cracked beam torsion problem $\cdot$ singularities $\cdot$ polygonal meshes

\section{Introduction}

Over the past sixty years, finite element methods involving the use of piecewise polynomial approximating functions have been extensively employed for solving many engineering problems with adequate accuracy, particularly in the fields of solid and structural mechanics. However, the use of piecewise polynomial functions is very inefficient in capturing solutions containing singularities [1], such as those typically encountered in fracture mechanics problems: see for instance, the crack tip problem in a fractured elastic body [2] or the torsion problem of a cracked prismatic beam [3]. Incorporating the form of the singularity in a numerical scheme is, generally, more effective than mesh refinement. Since the Seventies, many computational techniques have been proposed to tackle this issue in the finite element framework. Early methods successfully addressing the finite element solution of problems with singularities include both the singular basis function method [4] and the integrated singular basis function approach [5]. Among the more recent approaches, a prominent role is played by enriched approximations based on the partition-of-unity scheme introduced by Melenk and Babuška [6,7], such as the extended finite element method (X-FEM) [8-10].

First proposed in [11], the Virtual Element Method (VEM) is a recent development of stable Galerkin discretizations on polytopal meshes for boundary-value problems. Differently from the FEM, in the VEM, the shape functions are defined as the solution of a local elliptic partial differential equation and are never explic- 
itly computed. Hence, the finite element space spanned by such virtual shape functions is known as virtual element space. Since virtual shape functions are unknown, the VEM entails the construction of the approximation of the bilinear form by means of suitable elliptic polynomial projections, which are computable from the degrees of freedom of the method. In particular, the approximated bilinear form is composed of two parts: the consistency term, which approximates the stiffness matrix on a given polynomial field, and a correction term ensuring stability.

Like the FEM, the VEM requires a special treatment in order to satisfactorily approximate singular solutions. For instance, similarly to the hp-version of FEM, Beirão da Veiga et al. [12] proposed the use of geometrically refined meshes with appropriate local degree of accuracy to study L-shaped domains with geometric singularities. Moreover, Nguyen et al. [13] devised a VEM methodology for the modeling of crack propagation in 2D for linear elastic fracture, which places the crack line along the inter-element edges and exploits an adaptive mesh refinement procedure based on superconvergent patch recovery.

By generalizing the main concepts from the X-FEM, the Authors have recently contributed to formulating the etended virtual element method (X-VEM) for twodimensional problems governed by the Laplace equation in the presence of both singularities and strong discontinuities [14]. The basic idea of the X-VEM for problems with singularities is to enrich the standard virtual element space by means of an additional set of shape functions built upon suitably chosen enrichment functions that accurately describe the behavior of the solution near to the singularity. The choice of enriching the virtual element space was previously investigated in the plane wave virtual element method developed by Perugia et al. [15], who exploited approximations spaces made of products of functions that constitute a partition of unity and plane waves. However, to the Authors' knowledge, the proposed X-VEM represents the first attempt of exploiting the flexibility of the VEM for the construction of efficient approximations on general finite element meshes in the presence of singularities in the primal field [14].

In particular, in the X-VEM, the standard VEM projection operator has been modified to project functions onto an extended polynomial space spanned by standard basis monomials and one ore more enrichment functions. In [14], the X-VEM has been tested on the Laplace problem for an L-shaped domain, whose solution exhibits a singularity at the re-entrant corner, and the exact global solution was chosen as enrichment function. Since the enrichment coincides with the exact solution, the extended projection has been applied to every element of the mesh, observing a large improvement in both accuracy and convergence rate, which approached the optimal rate predicted by theory.

In the present paper, we assess the capability of the $\mathrm{X}-\mathrm{VEM}$ previously proposed in [14] to accurately approximate the problem of the torsion of a prismatic cracked beam, which is described by the Poisson equation in terms of a two-dimensional scalar stress function. This problem is especially meaningful in that it preludes to the general crack tip problem in fractured elastic continua. For the cracked beam torsion problem, no exact global solution is available as an analytic expression. However, it is known that the solution has a singularity on the crack tip, usually placed at the origin of the coordinate system, and can be expanded in a trigonometric series on a neighboorhood of the origin [16]. Hence, we choose the leading term of the series as the enrichment function for the X-VEM. Moreover, a geometric enrichment around the singularity is adopted, meaning that all the nodes within a given radius from the singularity are enriched. Thus, we only use the extended projector on elements containing enriched nodes, whereas the standard projector is employed for the remaining non-enriched elements. We show that the X-VEM performs equally well in this configuration, by greatly improving both accuracy and convergence rates in comparison to standard FEM and VEM.

The paper is organized as follows. In Section 2, we discuss the problem of the cracked beam under torsion. In Section 3, we present the X-VEM for problems governed by the Laplace equation in the presence of singularities, while Section 4, describes the main aspects of the implementation of the method. Numerical results are presented and discussed in Section 5. Finally, Section 6 is devoted to final remarks and conclusions.

\section{The torsion problem for a cracked beam}

The classical theory of the torsion of prismatic beams is a milestone of the theory of elasticity. Its foundation can be ascribed to Barrè de Saint Venant [17] and owes important contributions to other prominent scientists [18].

In particular, let us consider the case of a cracked prismatic beam of square cross section $\Lambda$, with boundary $\partial \Lambda$, subjected to torsion, shown in Fig. 1. We introduce the coordinates system $(x, y)$ principal of inertia and centered at the barycenter of $\Lambda$. The problem is governed by the following Poisson equation in terms of the scalar Prandtl stress function $v$ with homogeneous 
boundary conditions:

$$
\begin{aligned}
\Delta v & =-F & & \text { in } \Lambda, \\
v & =0 & & \text { on } \partial \Lambda .
\end{aligned}
$$

In (1), $F=2 G \bar{\vartheta}$ where $G$ is the tangential elastic modulus and $\bar{\vartheta}$ is the unit torsion angle of the beam. The

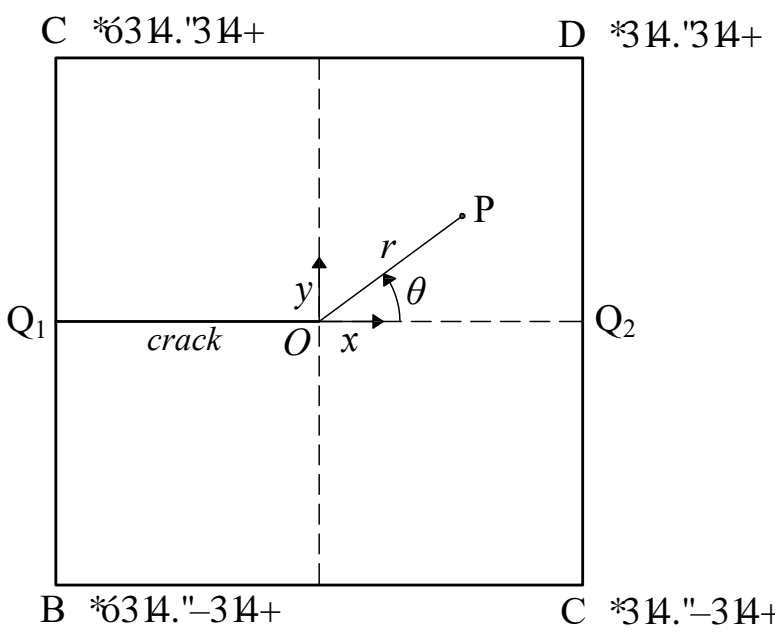

Fig. 1: Cracked square beam cross section.

solution $v$ of problem (1) is singular at the origin $O$ and can be expanded by a trigonometric series with leading term $r^{1 / 2} \cos \theta / 2$, where $(r, \theta)$ are the polar coordinates represented in Fig. 1. More precisely, it has been shown in $[16,5]$ that

$v=\sum_{j=1}^{\infty} \alpha_{j} r^{(2 j-1) / 2} \cos \left[\left(\frac{2 j-1}{2}\right) \theta\right]$

is analytic in a neighborhood of $O$ for suitable coefficients $\alpha_{j}$. Following the development in [4], in order to avoid physically uninteresting logarithmic singularities, boundary condition (1b) is replaced with:

$$
\begin{array}{rlrl}
v & =0 & & \text { on } \overline{Q_{1} O}, \overline{A D}, \text { and } \overline{B C}, \\
\frac{\partial v}{\partial n}=0 & \text { on } \overline{A B}, \overline{C D}
\end{array}
$$

where $\frac{\partial v}{\partial n}$ is the normal derivative to the boundary. As demonstrated in [16], the solution $v$ to (1a), (3a), (3b) has a singularity in $O$ of the form (2).

For the sake of simplicity, we assume $F=1$ and apply the transformation $u=v+y^{2} / 2$ so that equation (1a) together with boundary conditions (3a), (3b) can be rewritten as a Laplace equation in terms of the new variable $u$ as follows:

$$
\begin{aligned}
\Delta u & =0 & & \text { in } \Lambda, \\
u & =\frac{y^{2}}{2} & & \text { on } \overline{Q_{1} O}, \overline{A D}, \text { and } \overline{B C}, \\
\frac{\partial u}{\partial n} & =0 & & \text { on } \overline{A B}, \overline{C D} .
\end{aligned}
$$

Finally, we appeal to the symmetry to restrict problem (4a)-(4c) to the rectangle $\Omega \subset \Lambda$ with boundary $\partial \Omega$, shown in Fig. 2 .

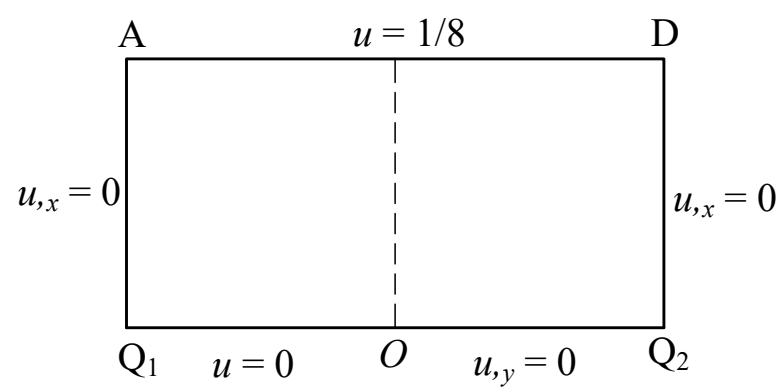

Fig. 2: Symmetrized region.

Be $\Gamma_{D}$ and $\Gamma_{N}$ the parts of $\partial \Omega$ where Dirichlet and Neumann boundary conditions are given, respectively. In order to define the weak form of problem (4), let $V_{0}=H_{0}^{1}(\Omega)$ denote the linear subspace of functions in the Sobolev space $H^{1}(\Omega)$ whose trace on $\Gamma_{D}$ is equal to zero. Furthermore, be $V=H_{\partial \Omega}^{1}(\Omega)$ the affine subspace of functions in $H^{1}(\Omega)$ whose restriction on $\Gamma_{D}$ is equal to $y^{2} / 2$. The variational formulation of problem (4a)(4c) reads as: find $u \in V$ such that

$a(u, v)=0 \quad \forall v \in V_{0}$,

where the bilinear form $a(\cdot, \cdot)$ is given by

$a(u, v)=\int_{\Omega} \nabla u \cdot \nabla v d \boldsymbol{x}$.

Noteworthy, the Dirichlet boundary condition in (4b) is incorporated in the definition of the functional space $V$.

\section{Extended virtual element approximation}

In this Section, we closely follow the procedural outline presented in [14]. For the sake of conciseness, we will hereafter provide the essential aspects of the method and refer the reader to [14] for a thorough presentation of the general ideas behind the X-VEM. 
From the weak form of the continuous problem (5), we can state the extended virtual element discrete problem as: find $u_{X}^{h} \in V_{X, \partial \Omega}^{h} \subset V$ such that

$a_{h}\left(u_{X}^{h}, v_{X}^{h}\right)=0 \quad \forall v_{X}^{h} \in V_{X, 0}^{h} \subset V_{0}$,

where $a_{h}(\cdot, \cdot)$ is the virtual element bilinear form that approximates the exact bilinear form $a(\cdot, \cdot)$. Spaces $V_{X, \partial \Omega}^{h}$ and $V_{X, 0}^{h}$ are built from the enrichment of the standard finite-dimensional conforming virtual element space $V^{h} \in$ $V$. Let $\left\{\phi_{i}\right\}_{i=1}^{N}$ be the set of canonical X-VEM basis functions, deriving from the discretization of the problem domain $\Omega$. After expanding the trial and test functions as a linear combination of these basis functions and substituting them in (7), owing to the arbitrariness of the test coefficients, we obtain the following linear system of equations:

$\boldsymbol{K} \boldsymbol{d}=\mathbf{0}, \quad K_{i j}=a_{h}\left(\phi_{i}, \phi_{j}\right)$,

where $\boldsymbol{K}$ is the stiffness matrix and $\boldsymbol{d}$ is the unknown coefficients vector. We consider first-order virtual elements. Therefore, we define the space $\mathbb{P}_{1}(\Omega)$ of linear polynomials on $\Omega$. For each element $E$, we introduce the local virtual element space $V^{h}(E)$, which includes the linear polynomials $\mathbb{P}_{1}(E)=\operatorname{span}\{1, x, y\}$ as a subspace. Moreover, we define the global virtual element space $V^{h}$ on $\Omega$. Any virtual element function $v^{h}$ in $V^{h}(E)$ is uniquely determined by its vertex values, known as the degrees of freedom (DOFs), from which it is possible to compute the elliptic projection $\Pi^{\nabla} v^{h}$ onto linear polynomials. We now introduce the enriched local and global virtual element spaces for the X-VEM, i.e., $V_{X}^{h}(E)$ and $V_{X}^{h}$, incorporating additional information about the behavior of the singular solution by means of a suitable enrichment function denoted by $\psi$. We also define the more general projection operator $\Pi_{X}^{\nabla} v_{X}^{h}$ onto $\operatorname{span}\{1, x, y, \psi\}$, which allows to construct the extended virtual element bilinear form $a_{h}(\cdot, \cdot)$ used in (7).

\subsection{Standard virtual element space}

Let the mesh be a family $\mathcal{T}=\left\{\Omega_{h}\right\}_{h}$ of decompositions of $\Omega$ into nonoverlapping polygonal elements $E$, with nonintersecting boundary $\partial E$ and straight edges. Be $\boldsymbol{x}_{E}:=\left(x_{E}, y_{E}\right)$ and $h_{E}=\sup _{\boldsymbol{x}, \boldsymbol{y} \in E}|\boldsymbol{x}-\boldsymbol{y}|$ the barycenter and diameter of element $E$, respectively. Moreover, be $N_{E}$ the number of vertices of element $E$, oriented in counter-clockwise order and let $\boldsymbol{x}_{k}:=\left(x_{k}, y_{k}\right), k=$ $1,2, \ldots, N_{E}$ denote their coordinates. We finally indicate by $\boldsymbol{n}_{E, e}$ the unit outward normal vector to edge $e \in \partial E$.
Let the standard local virtual element space on each polygonal element $E$ with boundary $\partial E$ be defined as:

$$
\begin{array}{r}
V^{h}(E)=\left\{v^{h} \in H^{1}(E): \Delta v^{h}=0,\right. \\
\left.\left.v^{h}\right|_{\partial E} \in C^{0}(\partial E),\left.v^{h}\right|_{e} \in \mathbb{P}_{1}(e) \forall e \in \partial E\right\} .
\end{array}
$$

The space $V^{h}(E)$ contains all harmonic functions defined on $E$ whose restriction on the elemental boundary $\partial E$ is a continuous piecewise-linear polynomial. It follows from the definition (9) that the space of linear polynomials on $E, \mathbb{P}_{1}(E)$, is a subspace of $V^{h}(E)$. A basis for $\mathbb{P}_{1}(E)$ can be constructed from the following set of scaled monomials:

$m_{1}(\boldsymbol{x})=1, \quad m_{2}(\boldsymbol{x})=\frac{x-x_{E}}{h_{E}}, \quad m_{3}(\boldsymbol{x})=\frac{y-y_{E}}{h_{E}}$,

so that $\mathbb{P}_{1}(E)=\operatorname{span}\left\{m_{1}, m_{2}, m_{3}\right\}$. Since any virtual element function $v^{h} \in V^{h}(E)$ is uniquely determined by the degrees of freedom of $v^{h}$, we can compute the elliptic projection operator $\Pi^{\nabla}: V^{h}(E) \rightarrow \mathbb{P}_{1}(E)$, which, for $v^{h} \in V^{h}(E)$, is defined by the following condition [11]:

$$
\int_{E} \nabla \Pi^{\nabla} v^{h} \cdot \nabla q d \boldsymbol{x}=\int_{E} \nabla v^{h} \cdot \nabla q d \boldsymbol{x} \quad \forall q \in \mathbb{P}_{1}(E),
$$

with the additional requirement

$$
\int_{\partial E}\left(\Pi^{\nabla} v^{h}-v^{h}\right) d s=0
$$

Integrating by parts and recalling that linear polynomials are harmonic functions yields:

$$
\int_{E} \nabla \Pi^{\nabla} v^{h} \cdot \nabla q d \boldsymbol{x}=\sum_{e \in \partial E} \int_{e} v^{h} \boldsymbol{n}_{e} \cdot \nabla q d s
$$

where the edge integrals are computable because the degrees of freedom of $v^{h}$ allow us to uniquely determine the restriction of $v^{h}$ on each edge through linear interpolation. Equation (13) makes it possible to compute the elliptic projection by means of a linear system of equations. The global conforming virtual element space $V^{h}$ is obtained by the union of all the local spaces $V^{h}(E)$ and is defined as:

$$
V^{h}:=\left\{v^{h} \in H^{1}(\Omega):\left.v^{h}\right|_{E} \in V^{h}(E) \forall E \in \Omega_{h}\right\} .
$$


3.2 Extended virtual element space and the elliptic projection

In order to provide an accurate virtual element solution of problem (4), we enrich the local standard virtual element space with the harmonic function $\psi=r^{1 / 2} \cos \theta / 2$ on the polygonal element $E$, so that the extended virtual element space can be defined as:

$V_{X}^{h}(E):=V^{h}(E)+\psi V^{h}(E)$.

In order to construct the enriched bilinear form $a_{h}$, we need to define the extended elliptic projection operator $\Pi_{X}^{\nabla}: V_{X}^{h}(E) \rightarrow \mathbb{P}_{1,0}^{X}=\operatorname{span}(1, x, y, \psi)$ for each element $E$. Analogously to (11) and (12), the extended elliptic projection $\Pi_{X}^{\nabla} v_{X}^{h}$ for a given $v_{X}^{h} \in V_{X}^{h}(E)$, is the solution of the variational problem:

$$
\begin{array}{r}
\int_{E} \nabla \Pi_{X}^{\nabla} v_{X}^{h} \cdot \nabla q_{X} d \boldsymbol{x}=\int_{E} \nabla v_{X}^{h} \cdot \nabla q_{X} d \boldsymbol{x} \\
\forall q_{X} \in \mathbb{P}_{1,0}^{X}(E),
\end{array}
$$

together with the condition:

$\int_{\partial E}\left(\Pi_{X}^{\nabla} v_{X}^{h}-v_{X}^{h}\right) d s=0$.

Similarly to standard VEM projection, the projection $\Pi_{X}^{\nabla} v_{X}^{h}$ onto the subspace $\mathbb{P}_{1,0}^{X}(E)$ is computable from the degrees of freedom of $v_{X}^{h}$. In fact, recalling that $q_{X}$ is harmonic, integration by part provides the following equality:

$\int_{E} \nabla \Pi_{X}^{\nabla} v_{X}^{h} \cdot \nabla q_{X} d \boldsymbol{x}=\sum_{e \in \partial E} \int_{e} v_{X}^{h} \boldsymbol{n}_{e} \cdot \nabla q_{X} d s$

which holds for any function $q_{X} \in \mathbb{P}_{1,0}^{X}(E)$. The edge integrals on $\partial E$ are computable since the restriction of $v_{X}^{h}$ can be obtained by linear interpolation of the nodal degrees of freedom.

\subsection{Bilinear form}

The construction of the X-VEM straightforwardly follows the scheme outlined for the standard VEM [11]. The global discrete bilinear form $a_{h}\left(u_{X}^{h}, v_{X}^{h}\right)$ for $u_{X}^{h}, v_{X}^{h} \in$ $V_{X}^{h}$ is defined as the sum of elemental contributions

$a_{h}\left(u_{X}^{h}, v_{X}^{h}\right)=\sum_{E \in \Omega_{h}} a_{h}^{E}\left(u_{X}^{h}, v_{X}^{h}\right)$

where each local bilinear form can be computed as

$$
\begin{array}{r}
a_{h}^{E}\left(u_{X}^{h}, v_{X}^{h}\right)=\int_{E} \nabla \Pi_{X}^{\nabla} u_{X}^{h} \cdot \nabla \Pi_{X}^{\nabla} v_{X}^{h} d \boldsymbol{x} \\
+S^{E}\left(\left(I-\Pi_{X}^{\nabla}\right) u_{X}^{h},\left(I-\Pi_{X}^{\nabla}\right) v_{X}^{h}\right) .
\end{array}
$$

In (20), the first term on the right hand side is the socalled consistency term, which ensures the linear and $\psi$-consistency of the method, whereas $S^{E}(\cdot, \cdot)$ is known as stabilization term and guarantees the stability of the method. Different effective choices for the stabilization terms have been proposed in $[19,20]$.

\section{Notes on the implementation of the method}

Similarly to the FEM, any virtual element function of the local space $V^{h}(E)$ can be written as the Lagrange interpolation of the $N_{E}$ canonical basis functions $\varphi_{j}$ for $j=1, \ldots, N_{E}$, associated with the vertices $\boldsymbol{x}_{j}$ of $E$; in particular, each basis function $\varphi_{j}$ takes the value 1 at the $j$-th node $\boldsymbol{x}_{j}$ and zero at all the other nodes, and has compact support, the support region being the patch of elements that have node $\boldsymbol{x}_{j}$ in common. Let the mesh $\Omega_{h}$ be composed of $N$ nodes. Then, it follow immediately that the set of $N$ basis functions $\varphi_{j}$ in $\Omega_{h}$ is a partition-of-unity $(\mathrm{PU})$ on $\Omega_{h}$ :

$\sum_{j=1}^{N} \varphi_{j}(\boldsymbol{x})=1 \quad \forall \boldsymbol{x} \in \Omega_{h}$.

Any virtual element function of the extended space $V_{X}^{h}(E)$ can be expressed as:

$v_{X}^{h}(\boldsymbol{x})=\sum_{i \in \mathbb{I}} \varphi_{i}(\boldsymbol{x}) v_{i}^{0}+\sum_{j \in \mathbb{J} \subseteq \mathbb{I}} \psi(\boldsymbol{x}) \varphi_{j}(\boldsymbol{x}) v_{j}^{1} \quad \forall \boldsymbol{x} \in E$,

where $\mathbb{I}$ is an index set containing the nodes of the element $E$, whereas the set $\mathbb{J} \in \mathbb{I}$ indexes the enriched nodes. Finally, $v_{j}^{0}$ and $v_{j}^{1}$ are the nodal degrees of freedom of the standard virtual element functions $v_{0}^{h}$ and $v_{1}^{h}$.

4.1 Matrix representation of the elliptic projector $\Pi_{X}^{\nabla}$

We build the local stiffness matrix $\boldsymbol{K}^{E}$ from the extended projection operator $\Pi_{X}^{\nabla}$ mapping the enriched virtual function $v_{X}^{h}$ onto linear monomials and $\psi$. Under the assumption that all the element nodes are enriched and making use of the set of Lagrange basis functions previously defined, we represent the projection operator $\Pi_{X}^{\nabla}$ defined on $E$ as a $4 \times 2 N_{E}$ matrix $\Pi^{X}$. The $j$-th column of $\boldsymbol{\Pi}^{X}$ contains the coefficients $\left(\boldsymbol{a}_{j}^{X}\right)^{T}=$ $\left\{a_{1 j}^{X}, a_{2 j}^{X}, a_{3 j}^{X}, a_{4 j}^{X}\right\}^{T}$ of $\Pi_{X}^{\nabla} \phi_{j}$, projection of the $j$-th basis function $\phi_{j}$ on the scaled monomials and $\psi / \psi_{0}$. Let $m_{i}^{X}$ for $i=1, \ldots, 4$ be the scaled functions that form a basis for the extended space $\mathbb{P}_{1,0}^{X}=\mathbb{P}_{1}(E)+\psi \mathbb{P}_{0}(E)$. In particular, $m_{i}^{X}$ for $i=1,2,3$ are the scaled monomials and $m_{4}^{X}=\psi / \psi_{0}$ is the scaled enriched function. It 
is possible to compute the matrix $\boldsymbol{\Pi}^{X}$ by solving the linear system

$\boldsymbol{G}^{X} \boldsymbol{\Pi}^{X}=\boldsymbol{B}^{X}$

where the components $G_{i j}^{X}$ of $\boldsymbol{G}^{X}$ are given by

$G_{i j}^{X}= \begin{cases}\frac{1}{N_{E}} \sum_{k=1}^{N_{E}} m_{j}^{X}\left(\boldsymbol{x}_{k}\right) & \text { for } i=1, \\ \int_{E} \nabla m_{i}^{X}(\boldsymbol{x}) \cdot \nabla m_{j}^{X}(\boldsymbol{x}) d \boldsymbol{x} & \text { for } i>1,\end{cases}$

where $j=1, \ldots, 4$, and the matrix $\boldsymbol{B}^{X}=\left(B_{i j}^{X}\right)$ is given by

$B_{i j}^{X}= \begin{cases}\frac{1}{N_{E}} \sum_{k=1}^{N_{E}} \phi_{j}\left(\boldsymbol{x}_{k}\right) & \text { for } i=1, \\ \int_{E} \nabla m_{i}^{X}(\boldsymbol{x}) \cdot \nabla \phi_{j}(\boldsymbol{x}) d \boldsymbol{x} & \text { for } i>1,\end{cases}$

where $j=1, \ldots, 2 N_{E}$. The matrix components $G_{i j}^{X}$ and $B_{i j}^{X}$ defined in (24) and (25) are readily computed as the corresponding integrals of the standard VEM [21].

\subsection{Enriched stiffness matrix}

As anticipated, the stiffness matrix $\boldsymbol{K}^{E}:=\left(K_{i j}^{E}\right)$ of the $\mathrm{X}-\mathrm{VEM}$ is constructed as the sum of a consistent part $\boldsymbol{K}^{c}$ and a stabilization part $\boldsymbol{K}^{s}$. On using (20), the entries of the stiffness matrix $\boldsymbol{K}^{E}$ are given by

$$
\begin{aligned}
K_{i j}^{E}=K_{i j}^{c} & +K_{i j}^{s}=\int_{E} \nabla \Pi_{X}^{\nabla} \phi_{i} \cdot \nabla \Pi_{X}^{\nabla} \phi_{j} d \boldsymbol{x} \\
& +S^{E}\left(\left(I-\Pi_{X}^{\nabla}\right) \phi_{i},\left(I-\Pi_{X}^{\nabla}\right) \phi_{j}\right),
\end{aligned}
$$

where the stabilization term is computed as

$$
\begin{aligned}
& S^{E}\left(\left(I-\Pi_{X}^{\nabla}\right) \phi_{i},\left(I-\Pi_{X}^{\nabla}\right) \phi_{j}\right)= \\
& =\alpha \operatorname{trace}\left(\boldsymbol{K}^{c}\right) \sum_{\ell=1}^{N_{E}} \operatorname{dof}_{\ell}\left[\left(I-\Pi_{X}^{\nabla}\right) \phi_{i}\right] \operatorname{dof}_{\ell}\left[\left(I-\Pi_{X}^{\nabla}\right) \phi_{j}\right],
\end{aligned}
$$

$\alpha$ being a scalar stabilization parameter and $\operatorname{dof}_{\ell}(\cdot)$ the functional that returns the value of the $\ell$-th degree of freedom when applied to a virtual element function.

\subsection{Numerical integration}

An enhanced quadrature scheme, especially conceived for singular functions, has been adopted for the computations of integrals in the matrix $G$ defined in (24), in the stiffness matrix entries $K_{i j}^{E}$ defined in (26), and strain energy computations as well. Polynomial approximations of such integrals are poor and require a huge number of quadrature points to attain sufficient accuracy. The enhanced quadrature scheme, hereafter referred to by the acronym HNI, is based on the numerical integration of homogeneous functions on convex and nonconvex polygons, developed by Chin et al. [22]. The HNI scheme allows to reduce integration of homogeneous functions over arbitrary polygons to integration over the boundary edges of the polygon. By definition, a positively homogeneous function of degree $q$ satisfies

$f(\lambda \boldsymbol{x})=\lambda^{q} f(\boldsymbol{x})$

for all $\boldsymbol{x}$ and where $\lambda>0$. Given a polygonal domain $M$ with edges $e_{i}(i=1, \ldots, m)$, let $\boldsymbol{a}_{i} \cdot \boldsymbol{x}=b_{i}$ the line containing edge $e_{i}$, where the sign of $b_{i}$ is determined such that $\boldsymbol{a}_{i} /\left\|\boldsymbol{a}_{i}\right\|$ is the outward normal to the polygon. Starting from definition (27), applying Euler's homogeneous function theorem and Stokes theorem, and finally using an $n q$-th point Guass quadrature rule, it is possible to prove that:

$\int_{M} f(\boldsymbol{x}) d \boldsymbol{x}=\frac{1}{2+q} \sum_{i=1}^{m} \frac{b_{i}}{\left\|\boldsymbol{a}_{i}\right\|} \sum_{j=1}^{n q} w_{i j} f\left(\boldsymbol{x}_{i j}\right)$.

With Gauss quadrature applied to the bounding line segments, (28) provides numerical integration with polynomial precision over arbitrary polygons. To improve the distribution of integration points required on each line segment, we adopt an adaptive integration scheme that provides an optimized, custom quadrature rule for each element, which allows to compute the integrals to a user-specified precision through an a posteriori estimation of the error. The reader is addressed to [23] for further details.

\section{Numerical results}

\subsection{The cracked beam torsion problem}

We present a convergence study for the X-VEM described in the previous sections applied to the cracked beam torsion problem (4), which, as discussed in Section 3, exhibits a singular solution at the crack tip. Recalling that the transformation $v=u-y^{2} / 2$ holds, from the solution $u$ of problem (4), the solution in terms of stress function $v$ of the original problem can be determined. The main features of the problem at hand, together with adopted geometry and boundary conditions, have been described in Section 3. In the X-FEM framework, it has been shown by Laborde et al. [24] and Béchet et al. [25] that optimal convergence is recovered if all nodes that lay within a fixed radius around the singularity are enriched. This strategy is referred to as geometric enrichment. Also for the X-VEM, it has 
been proved that geometric enrichment in the presence of singularities is beneficial to the convergence rate of the method [14]. For this reason, we apply geometric enrichment to the cracked beam torsion problem, by enriching all the nodes within a radius $r_{e}=0.2$ from the origin, where the singularity lays. Fig. 3 represents geometric enrichment for a mesh of quadrilateral elements. Since, in this case, the enrichment function does not coincide with the global exact solution of the problem, we only use the extended projector $\Pi_{X}^{\nabla}$ on elements containing enriched nodes, whereas the standard projector $\Pi^{\nabla}$ is employed for the remaining non-enriched elements. The convergence study has been conducted by means of two different sets of 5 meshes each. The first set is composed of $8,32,128,512,2048$ quadrilateral elements, respectively, while the second set is made of $8,28,88,300,1120$ polygonal elements, respectively; see Fig. 4 for an example of polygonal mesh. The number of elements in polygonal meshes has been chosen so that each polygonal mesh has the same number of degrees of freedom as the corresponding quadrilateral mesh. In particular, polygonal meshes have been generated starting from a Voronoi tassellation obtained with the Lloyd's algorithm [26]. To illustrate the strengths of the method, we compare the results obtained from the $\mathrm{X}-\mathrm{VEM}$ with results obtained from classic VEM and FEM. From an engineering point of view, the most in-

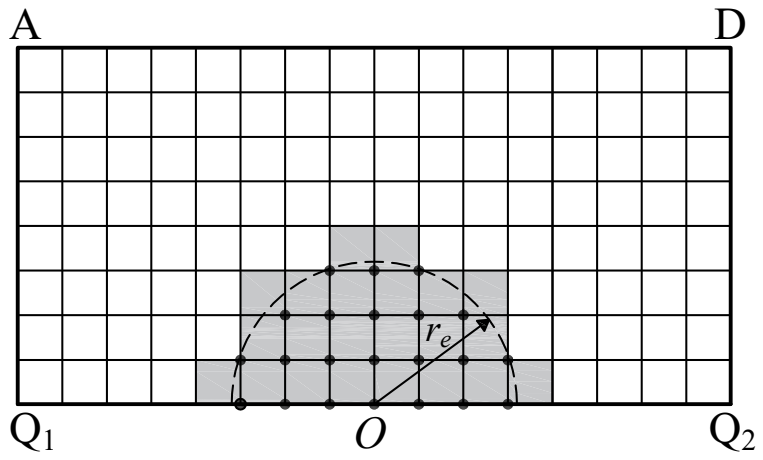

Fig. 3: Geometric enrichment in the cracked beam torsion problem. Nodes lying within the circle of radius $r_{e}=0.2$ are enriched.

teresting quantity is not the value taken by the stress function $v$ but, rather, the so-called stress intensity factor $\sigma_{0}$ defined as [4]:

$\sigma_{0}=\lim _{r \rightarrow 0} r^{-1 / 2}[v(r, 0)-v(0,0)]$,

which is a commonly accepted measure of the amount of torsion the beam can sustain before fracture takes place. Such quantity can be approximated from the numerical solution in terms of the stress function $v$ by

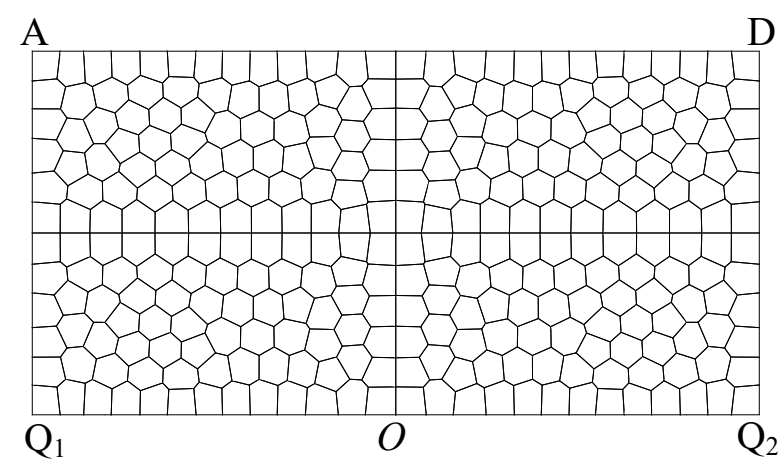

Fig. 4: Example of polygonal mesh.

means of the difference quotient [3]:

$\sigma_{0}=\frac{v_{h}\left(\xi_{h}, 0\right)-v_{h}(0,0)}{\xi_{h}^{1 / 2}}$

where $\xi_{h}$ is chosen such that $\xi_{h}=O(h)$, being $h$ a mesh dimension parameter. The exact value of the stress intensity factor for the cracked beam torsion problem is $\sigma_{0}=0.19112$ [5]. Fig. 5 displays convergence plots in terms of stress intensity factor at the crack tip for the XVEM and compares it to classic VEM and FEM. It can be drawn that, while both the FEM and VEM exhibit a poor convergence behavior, the X-VEM has a higher, steady convergence rate which guarantees significantly more accurate results. It is also interesting to study

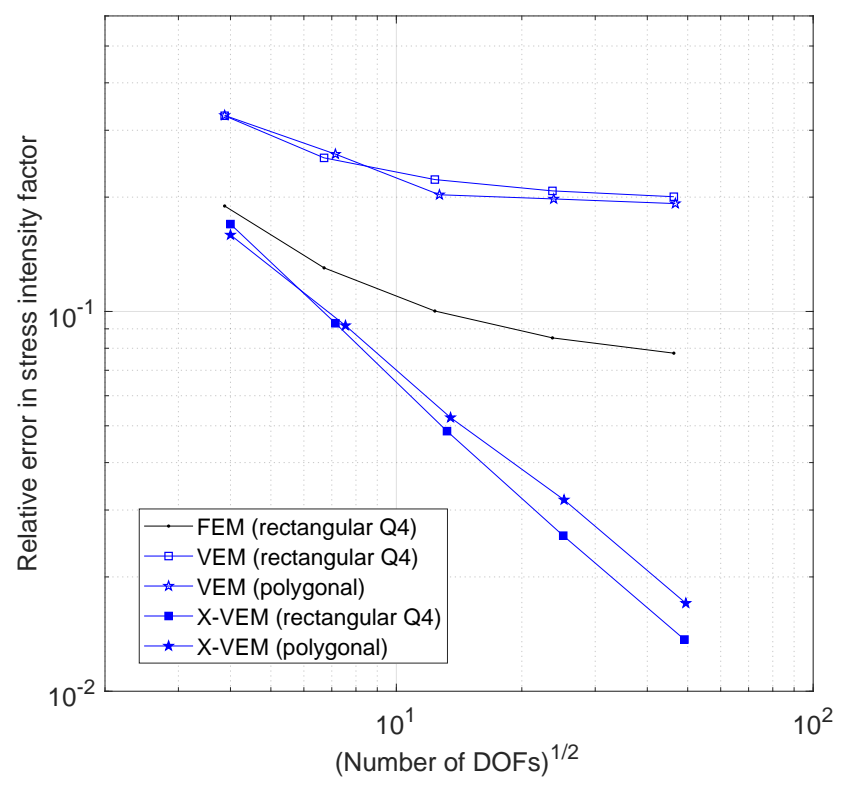

Fig. 5: Convergence in terms of stress intensity factor at the crack tip for the cracked beam torsion problem. X-VEM computations are done with the elliptic projector $\Pi_{X}^{\nabla}$ on quadrilateral and polygonal meshes and are compared to both the standard VEM and FEM 
the convergence of the approximate solution in terms of point values taken by stress function $v$ at three different locations in the problem domain [4]. In particular, points $P_{1} \equiv(0,1 / 24), P_{2} \equiv(-11 / 24,1 / 4)$ and $P_{3} \equiv$ $(11 / 24,1 / 4)$ have been considered, where the exact solution as been computed respectively as $v_{1}=0.027425$, $v_{2}=0.032877$ and $v_{3}=0.070844$. As shown in Figs. 6,7 and 8 , the X-VEM provides faster convergence and better accuracy than both FEM and VEM. Finally, con-

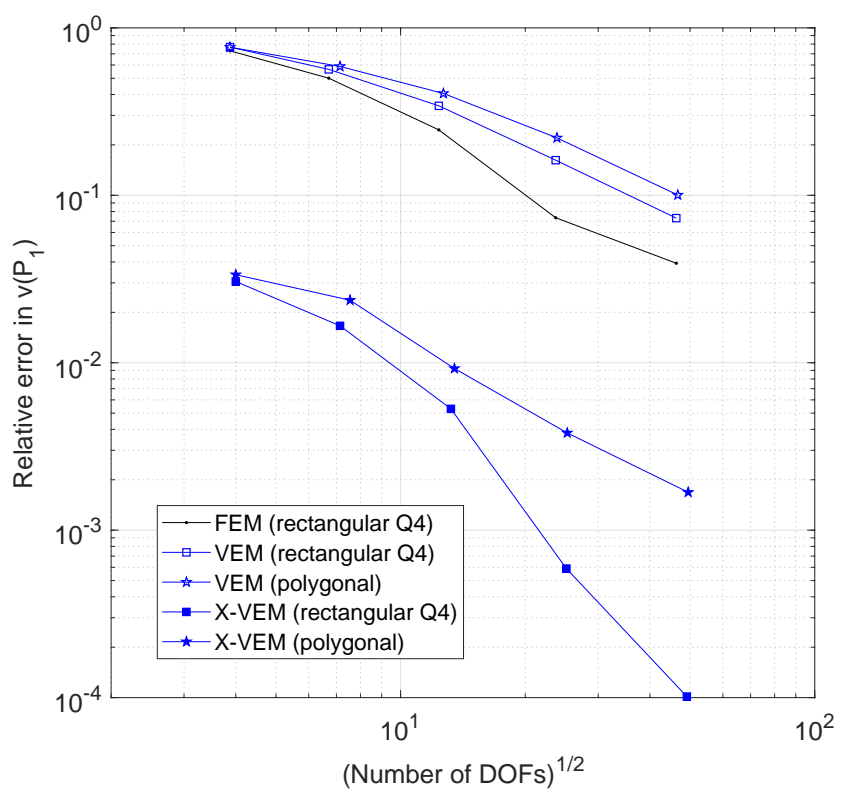

Fig. 6: Convergence in terms of stress function evaluated in $P_{1}$ for the cracked beam torsion problem. X-VEM computations are done with the elliptic projector $\Pi_{X}^{\nabla}$ on quadrilateral and polygonal meshes and are compared to both the standard VEM and FEM

vergence in relative error in strain energy has been investigated. Strain energy for the Laplace problem at hand is defined as [27]:

$E(v)=\frac{1}{2} \int_{\Omega} \nabla v \cdot \nabla v d V$.

Therefore, relative error in strain energy is:

$\epsilon_{r e l}=\frac{\left|E\left(\tilde{v}_{h}\right)-E\left(v_{e x}\right)\right|}{E\left(v_{e x}\right)}$,

where $v_{e x}$ is the exact solution and $\tilde{v}_{h}$ is the projection of the discrete solution $v_{h}$, defined as:

$\tilde{v}_{h}=\sum_{K \in \mathcal{T}} \Pi_{K}^{\nabla} v_{h}$

with $\Pi_{K}^{\nabla}=\Pi^{\nabla}$ for non-enriched elements and $\Pi_{K}^{\nabla}=\Pi_{X}^{\nabla}$ for elements containing enriched nodes. The reason for

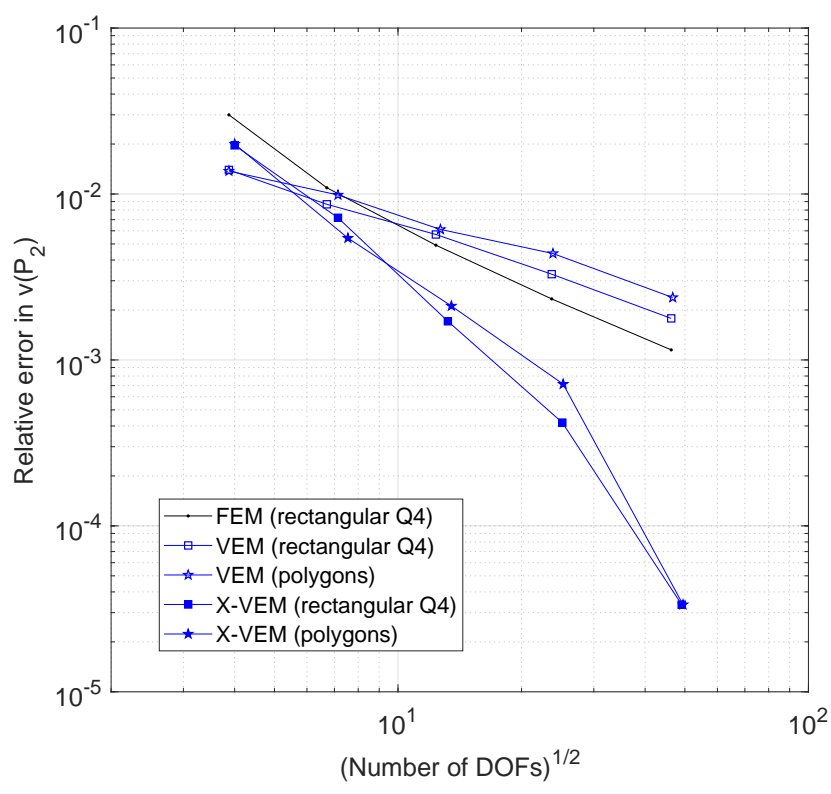

Fig. 7: Convergence in terms of stress function evaluated in $P_{2}$ for the cracked beam torsion problem. X-VEM computations are done with the elliptic projector $\Pi_{X}^{\nabla}$ on quadrilateral and polygonal meshes and are compared to both the standard VEM and FEM

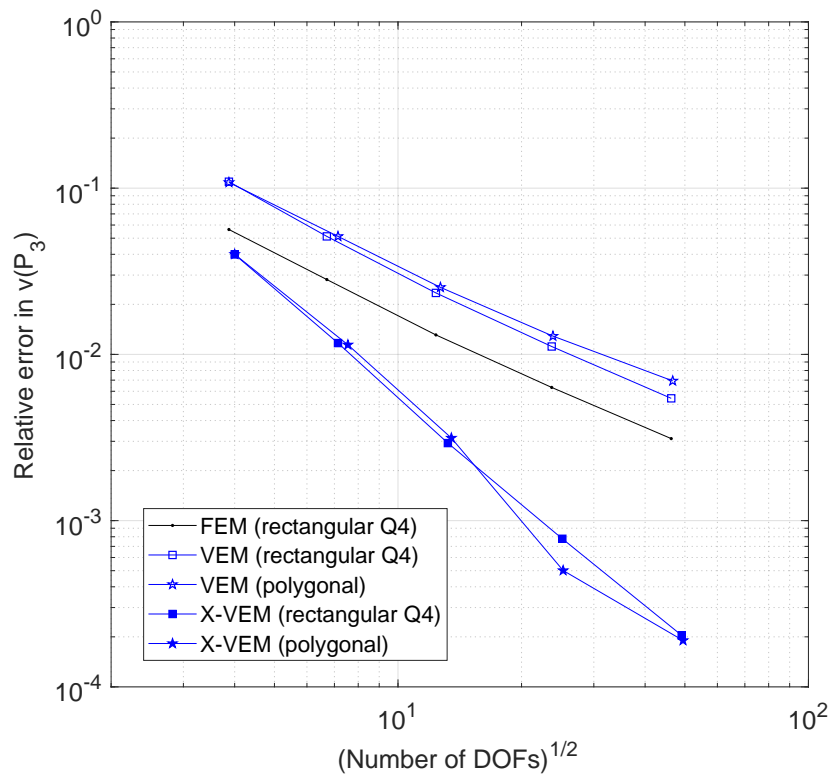

Fig. 8: Convergence in terms of stress function evaluated in $P_{3}$ for the cracked beam torsion problem. X-VEM computations are done with the elliptic projector $\Pi_{X}^{\nabla}$ on quadrilateral and polygonal meshes and are compared to both the standard VEM and FEM

the choice (33) is that it is not possible to compute the true energy associated to $v_{h}$, since virtual functions are not explicitly known [12]. Moreover, since the exact solution is not available as an analytic expression 
for the problem under study, it has been approximated by means of a higher order FEM solution, $v_{r e f}$, computed on an overkill mesh with 524288 elements. The corresponding strain energy, $E_{\text {ref }}=0.0097847$, is used in (32) in place of $E\left(v_{e x}\right)$. Fig. 9 depicts convergence plots in terms of relative error in strain energy for the $\mathrm{X}$-VEM, VEM and FEM respectively. Again, the XVEM provides a distinctive advantage in terms of accuracy and convergence rate over both the FEM and the VEM. In particular, the computed convergence rate in terms of relative error in strain energy is equal to 2.04 for the X-VEM and 1.18 for both the VEM and the FEM. Hence, the X-VEM allows to recover the optimal convergence rate in energy as predicted by theory for finite elements, which is equal to 2 . On the contrary, both VEM and FEM exhibit a suboptimal convergence rate of 1 , which is in agreement with theory as well: indeed, the theoretical asymptotic rate of convergence of finite element methods with polynomial degree $p$ for problems with a singularity of order $\lambda$ is $\min (2 p, 2 \lambda)$ [28] and, for the problem at hand, we have $p=1$ and $\lambda=1 / 2$.

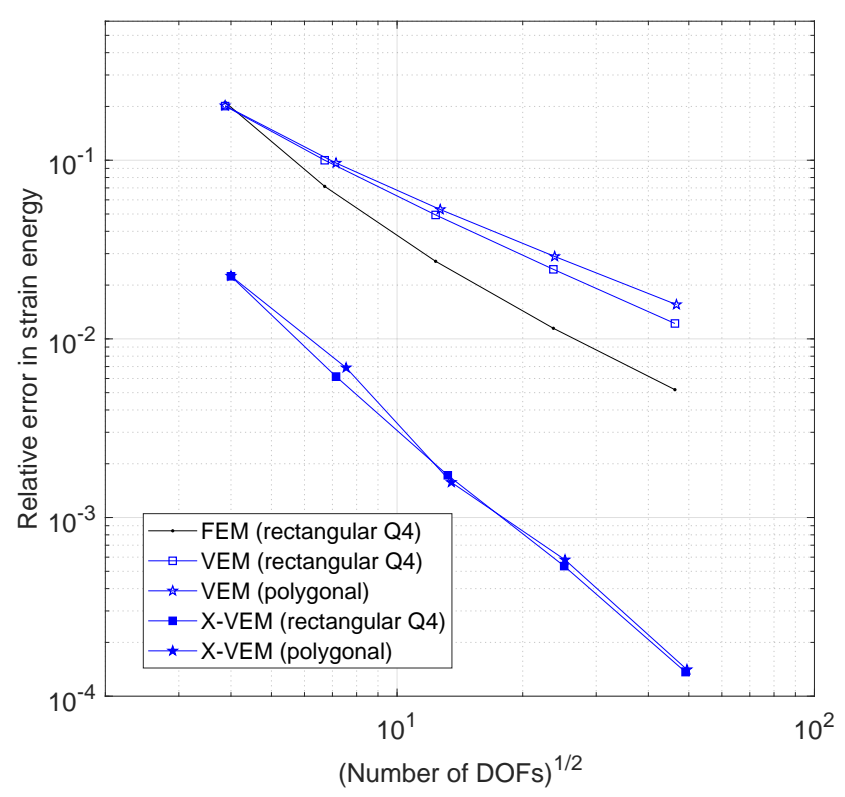

Fig. 9: Convergence in terms of strain energy for the cracked beam torsion problem. X-VEM computations are done with the elliptic projector $\Pi_{X}^{\nabla}$ on quadrilateral and polygonal meshes and are compared to both the standard VEM and FEM

\subsection{The Motz problem}

Very similar to the cracked beam torsion problem, the Motz problem [29] is a popular benchmark for testing the various approaches proposed in the literature for treating singularities. The problem is defined on the same square domain as the cracked beam torsion problem and its exact solution can be expanded in a neighborhood of the origin with the same trigonometric series (2). Fig. 10 depicts geometry and boundary conditions for the Motz problem as modified by Wait and Mitchell [30]. The convergence of the X-VEM on

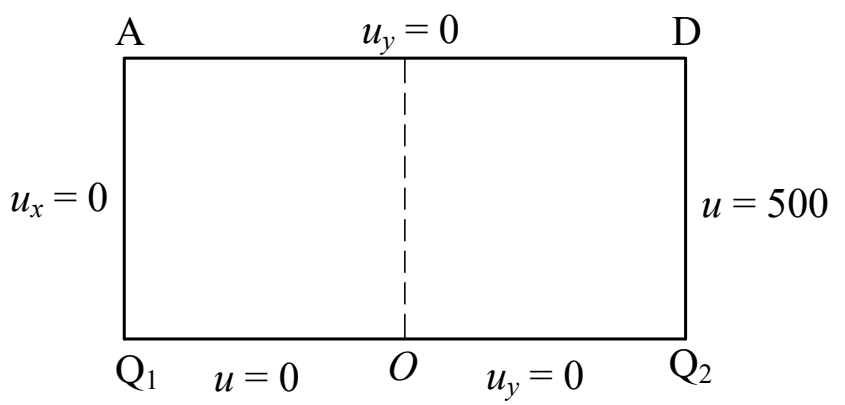

Fig. 10: Geometry and boundary conditions of the Motz problem

the Motz problem in terms of relative error in strain energy has been investigated. Since the exact global solution of the Motz problem is not known in closed form, the same approach adopted for the cracked beam torsion problem is here used to establish an accurate reference value for the strain energy, which has been computed as $E_{\text {ref }}=8.50795$. Fig. 11 shows convergence in terms of relative error in strain energy for the $\mathrm{X}$-VEM, VEM and FEM respectively. As in the case of the cracked beam under torsion, for the Motz problem, the X-VEM provides a definite advantage in terms of accuracy and convergence rate over standard FEM and VEM. In particular, the computed convergence rate in terms of relative error in strain energy is equal to 2.16 for the X-VEM and 1.05 for both the VEM and the FEM. Again, the X-VEM allows to recover the optimal convergence rate predicted by theory.

\section{Conclusions}

In this paper, we have presented the extended virtual element method (X-VEM), originally proposed in [14], for the torsion problem of a prismatic cracked beam, governed by the Poisson equation in terms of a two dimensional scalar stress function. This problem is characterized by a singularity in the primal field placed at 


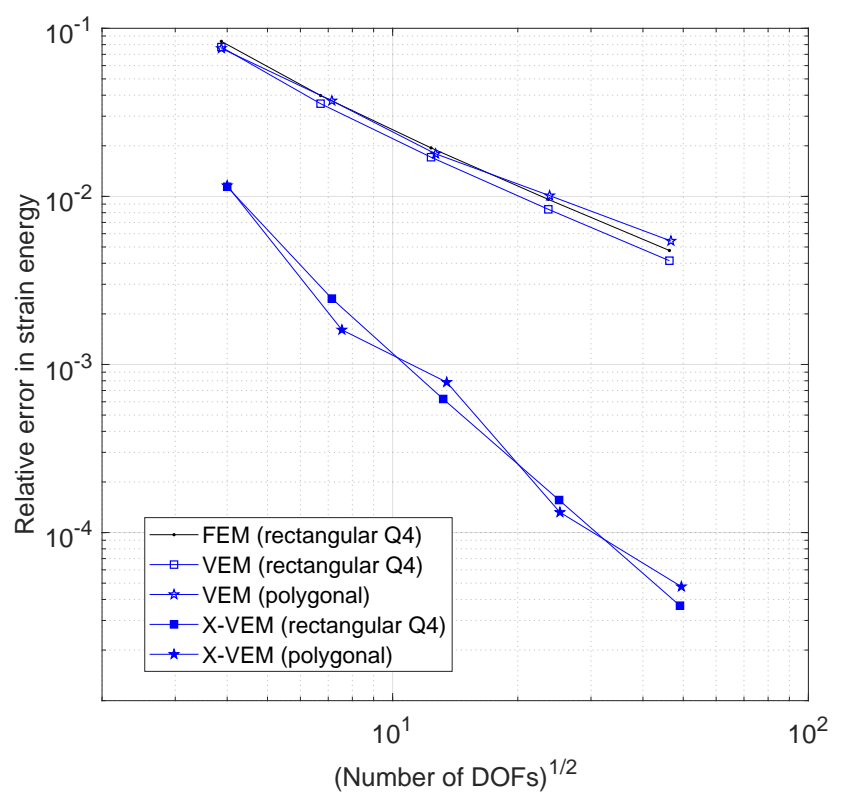

Fig. 11: Convergence in terms of strain energy for the Motz problem. X-VEM computations are done with the elliptic projector $\Pi_{X}^{\nabla}$ on quadrilateral and polygonal meshes and are compared to both the standard VEM and FEM

the crack tip. Even if no exact global solution is available as an analytic expression, an expansion in trigonometric series is available in a neighborhood of the origin. The leading term of the series has been taken as the enrichment function for the X-VEM and a geometric enrichment strategy around the singularity has been adopted, meaning that all the nodes within a given radius from the singularity have been enriched. Moreover, we have only used the extended projector on elements containing enriched nodes, whereas the standard projector has been employed for the remaining nonenriched elements. Through numerical experiments, we have investigated the convergence of the method in terms of stress intensity factor, stress function and strain energy. In all cases, we have shown that the X-VEM is more accurate than classic FEM and VEM. Moreover, $\mathrm{X}-\mathrm{VEM}$ allows to recover the expected optimal convergence rate.

Acknowledgements The Authors are grateful to Prof. N. Sukumar and Dr. G. Manzini for their valuable contributions to the developments contained in this paper.

\section{Funding}

This study was funded by Ministero dell'Istruzione, dell' Università e della Ricerca, PRIN: Progetti di Ricerca di Rilevante Interesse Nazionale (grant n. 2015LYYXA8).

\section{Conflict of interest}

The authors declare that they have no conflict of interest.

\section{References}

1. P. Grisvard, Elliptic Problems in Nonsmooth Domains, Pitman Publishing, Inc, Boston, MA, 1985.

2. G. Sih, P. Paris, F. Erdogan, Crack-tip, stress-intensity factors for plane extension and plate bending problems, Journal of Applied Mechanics 29 (2) (1962) 306-312.

3. W. Strang, G. Fix, An Analysis of the Finite Element Method, Prentice-Hall, Englewood Cliffs, NJ, 1973.

4. G. Fix, S. Gulati, G. Wakoff, On the use of singular functions with finite element approximations, Journal of Computational Physics 13 (1973) 209-228.

5. L. Olson, G. Georgiou, W. Schultz, An efficient finite element method for treating singularities in laplace's equation, Journal of Computational Physics 96 (1991) 391410.

6. J. M. Melenk, I. Babuška, The partition of unity finite element method: Basic theory and applications, Computer Methods in Applied Mechanics and Engineering 139 (1996) 289-314.

7. I. Babuška, J. M. Melenk, The partition of unity method, International Journal for Numerical Methods in Engineering 40 (1997) 727-758.

8. N. Moës, J. Dolbow, T. Belytschko, A finite element method for crack growth without remeshing, International Journal for Numerical Methods in Engineering 46 (1) (1999) 131-150.

9. N. Sukumar, J. E. Dolbow, N. Moës, Extended finite element method in computational fracture mechanics: a retrospective examination, International Journal of Fracture 196 (1) (2015) 189-206.

10. G. Ventura, E. Benvenuti, Equivalent polynomials for quadrature in heaviside function enriched elements, International Journal for Numerical Methods in Engineering 102 (3-4) (2015) 688-710.

11. L. Beirão da Veiga, F. Brezzi, A. Cangiani, G. Manzini, L. D. Marini, A. Russo, Basic principles of virtual element methods, Mathematical Models \& Methods in Applied Sciences 23 (2013) 119-214.

12. L. B. da Veiga, A. Chernov, L. Mascotto, A. Russo, Exponential convergence of the hp virtual element method in presence of corner singularities, Numerische Mathematik 138 (2018) 581-613.

13. V. M. Nguyen-Thanh, X. Zhuang, H. Nguyen-Xuan, T. Rabczuk, P. Wriggers, A Virtual Element Method for 2D linear elastic fracture analysis, Computer Methods in Applied Mechanics and Engineering 340 (2018) 366-395.

14. E. Benvenuti, A. Chiozzi, G. Manzini, N. Sukumar, Extended virtual element method for the laplace problem with singularities and discontinuities, Computer Methods in Applied Mechanics and Engineering 356 (2019) 571-597.

15. I. Perugia, P. Pietra, A. Russo, A plane wave virtual element method for the Helmholtz problem, ESAIM: Mathematical Modelling and Numerical Analysis 50 (3) (2016) 783-808.

16. S. Lehman, Developments at an analytic corner of solutions of elliptic partial differential equations, Journal of Mathematics and Mechanics 8 (1959) 727-760. 
17. A. Barré de Saint-Venant, De la torsion des prismes avec des considérations sur leurs flexion ainsi que sur l'équilibre des solides élastiques en général et des formules pratiques pour le calcul de leur résistance à divers efforts s'exerçant simultanément, Imprimerie, 1856.

18. L. Prandtl, Zur Torsion von Prismatischen Stäben, Zeitschrift für Physik 4 (1903) 758-770.

19. F. Dassi, L. Mascotto, Exploring high-order three dimensional virtual elements: Bases and stabilizations, Computers \& Mathematics with Applications 75 (9) (2018) 3379-3401.

20. L. Mascotto, Ill-conditioning in the virtual element method: Stabilizations and bases, Numerical Methods for Partial Differential Equations 34 (4) (2018) 1258-1281.

21. L. Beirão da Veiga, F. Brezzi, L. D. Marini, A. Russo, The hitchhiker's guide to the virtual element method, Mathematical Models \& Methods in Applied Sciences 24 (8) (2014) 1541-1573.

22. E. B. Chin, J. B. Lasserre, N. Sukumar, Numerical integration of homogeneous functions on convex and nonconvex polygons and polyhedra, Computational Mechanics 56 (6) (2015) 967-981.

23. E. B. Chin, J. B. Lasserre, N. Sukumar, Modeling crack discontinuities without element-partitioning in the extended finite element method, International Journal for Numerical Methods in Engineering 86 (11) (2017) 10211048.

24. P. Laborde, J. Pommier, Y. Renard, M. Salaün, Highorder extended finite element method for cracked domains, International Journal for Numerical Methods in Engineering 64 (3) (2005) 354-381.

25. E. Béchet, H. Minnebo, N. Moës, B. Burgardt, Improved implementation and robustness study of the X-FEM for stress analysis around cracks, International Journal for Numerical Methods in Engineering 64 (8) (2005) 10331056.

26. C. Talischi, G. Paulino, A. Pereira, F. Menezes, PolyMesher: a general-purpose mesh generator for polygonal elements written in Matlab, Structural and Multidisciplinary Optimization 45 (3) (2012) 309-328.

27. K. Bathe, Finite element procedures in engineering analysis, Prentice-Hall, Englewood Cliffs, NJ, 1982.

28. S. E. Mousavi, N. Sukumar, Generalized Duffy transformation for integrating vertex singularities, Computational Mechanics 45 (2-3) (2010) 127-140.

29. H. Motz, The treatment of singularities of partial differential equations by relaxation methods, Quarterly of Applied Mathematics 4 (4) (1947) 371-377.

30. R. Wait, A. Mitchell, Corner singularities in elliptic problems by finite element methods, Journal of Computational Physics 8 (1) (1971) 45-52. 